\title{
Oceanography
}

CITATION

Kaeli, J.W. 2017. Do AUVs dream of electric eels? Oceanography 30(2):169-171,

https://doi.org/10.5670/oceanog.2017.239.

$\mathrm{DOI}$

https://doi.org/10.5670/oceanog.2017.239

COPYRIGHT

This article has been published in Oceanography, Volume 30, Number 2, a quarterly journal of The Oceanography Society. Copyright 2017 by The Oceanography Society. All rights reserved.

USAGE

Permission is granted to copy this article for use in teaching and research.

Republication, systematic reproduction, or collective redistribution of any portion of this article by photocopy machine, reposting, or other means is permitted only with the approval of The Oceanography Society. Send all correspondence to: info@tos.org or The Oceanography Society, PO Box 1931, Rockville, MD 20849-1931, USA. 


\section{Do AUVs Dream of Electric Eels?}

By Jeffrey W. Kaeli

Free-swimming autonomous underwater vehicles (AUVs) are distinct from tethered remotely operated vehicles (ROVs) and human-occupied vehicles in the amount of data-driven feedback a human can provide during a mission. While free-space optical communications afford tether-equivalent data rates at relatively close ranges (Farr et al., 2010), most AUVs employ acoustic modems to maintain two-way communications with their operators while underway (Freitag et al., 2005). However, the low bandwidths and high latencies inherent in underwater acoustics prohibit the realtime transmission of data generated by imaging sensors such as cameras and side-scan sonars. This has profound implications with regard to the meaning of the data an AUV collects and the trust an operator has in the AUV's autonomy to react to data in the absence of direct human oversight.

Robots are fundamentally agnostic to the meaning of the data they collect, where meaning implies the qualitative ideas a human attaches to quantitative values. As more and more meaning is conveyed to the AUV, more and more trust can be built up by the operator. Thus, trust and meaning form a cyclic feedback loop that is throttled by the bandwidth limitations of underwater communication. Some of our recent work in marine autonomy at the Woods Hole Oceanographic Institution has focused on co-robotic frameworks that address this concept in the context of real-time AUV operations. This article briefly describes two such frameworks for summary-based mapping and anomaly detection that reinforce operator trust by enabling a human to impart meaning to data while they are still being collected by an AUV.

\section{SUMMARY-BASED MAPPING}

Online summaries (Girdhar and Dudek, 2010) are useful tools for data reduction in real time imaging pipelines, but their output lacks inherent meaning outside of a human's interpretation of the content. Compression algorithms now make transmitting imagery via acoustic modem during a mission a practical reality (Murphy and Singh, 2010), but they depend on a separate mechanism for selecting which images to transmit. Marrying these two strategies, the robot can first transmit a small number of images representing the classes that best characterize the data collected thus far, allowing a human to attribute meaning to them. Combining class membership of non-summary images with navigational metadata enables the creation of simple maps whose indices correspond to each of the summary classes (Kaeli and Singh, 2015). Figure 1 shows an example of this technique using 2,800 images collected by the SeaBED AUV in 2003 from the Stellwagen Bank Marine Sanctuary.
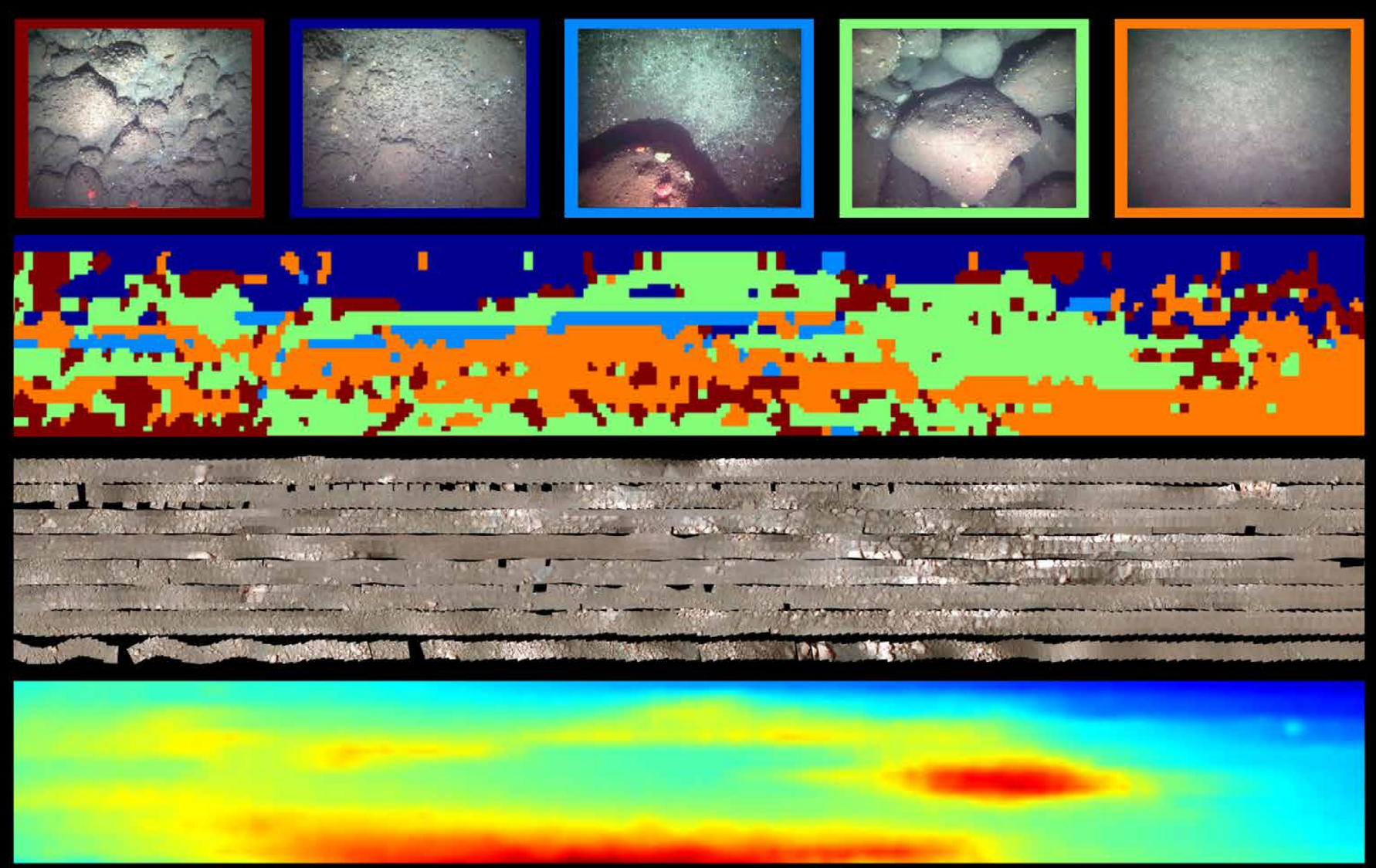

FIGURE 1. From to top to bottom: a set of five images the algorithm selected that best summarize the data set, a summary-based map color coded by image class, a photomosaic of the entire mission, and bathymetry of the survey area. While the robot only understands the data in the context of "Class 1" or "Class 2," a human is able to attribute meaning such as "rocky" or "sandy" to the images and relate that meaning to the broader context of habitat or geomorphology. 
Contrary to offline algorithms that require all data to be collected prior to analysis, online algorithms maintain a current summary of the data at any point in the mission. While they offer fewer guarantees of stability or finding the optimal solution, they do provide a practical basis from which an AUV can begin to adaptively sample its environment. Even in the absence of operator feedback, pre-set classes can be stored to enable the AUV to search for certain substrates, ignore entire areas, or transmit maps without the need for compressing imagery. At the end of a mission, the operator possesses both an immediate high-level summary of the survey and the ability to selectively download the most meaningful parts of the data set first. Enabling operators to begin interacting with data while a mission is underway makes the best use of both the AUV's resources and the operator's time while at sea.

\section{ANOMALY DETECTION}

A robot can be trained to automatically detect certain meaningful objects of interest, but many types of objects are difficult or impossible to characterize with prior training. In these cases, detecting anomalous regions that stand out from their surroundings can be useful for adaptively resurveying potential targets as opposed to relying on human post-mission analysis and a secondary deployment. We implement a two-tiered algorithm that first finds locally salient regions that differ from their surroundings, then clusters these regions to learn which are globally rare (Kaeli, 2016). Applied to side-scan sonar imagery collected during the search for Air France flight 447 (Figure 2), this approach detected several anomalous regions that include the wreckage of the airplane.
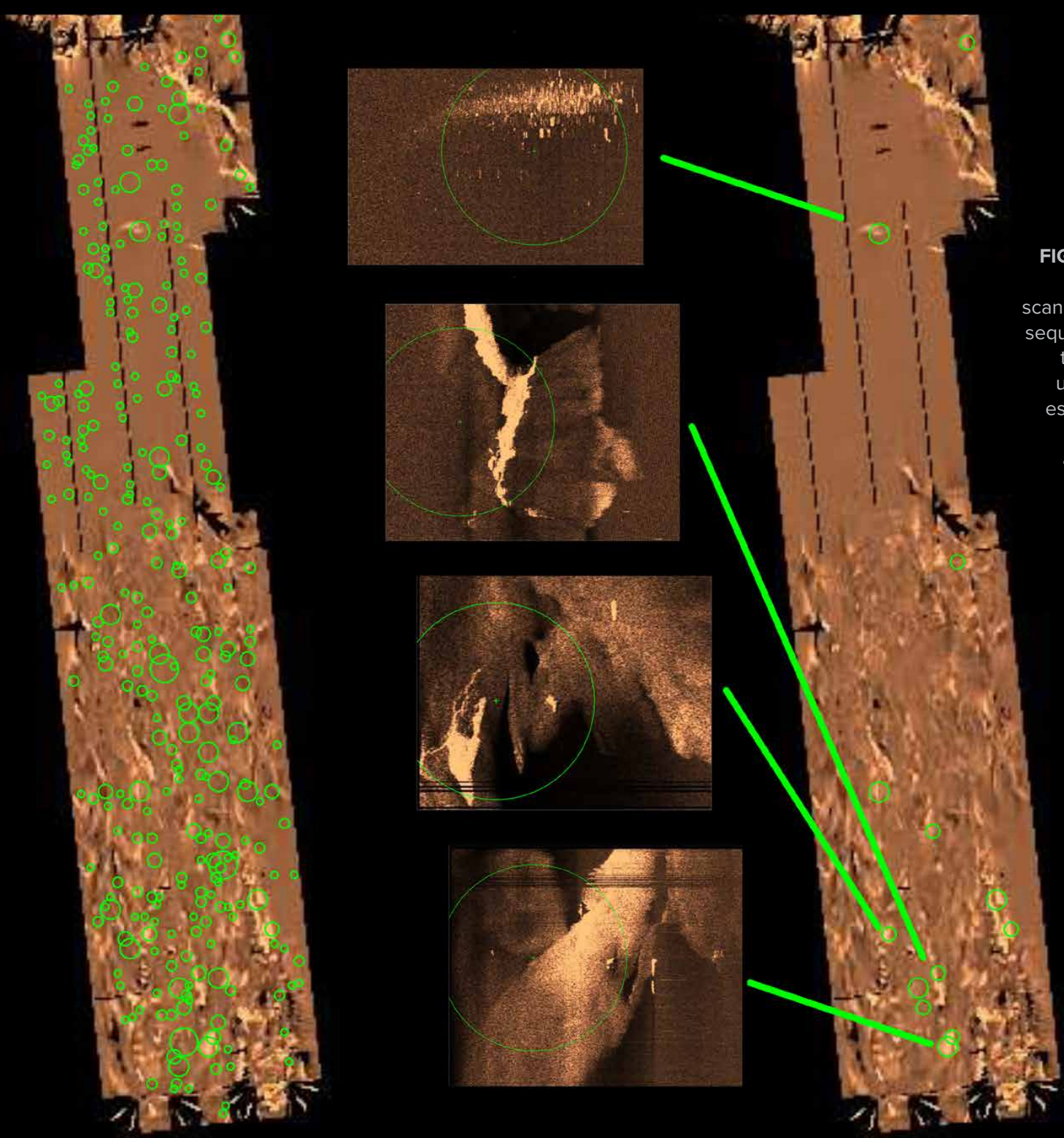

FIGURE 2. Locally anomalous regions detected in a sidescan sonar survey (left) are subsequently filtered to select only those that occur at a particular rarity and scale of interest (right). Sample detections (center) include the wreckage of Air France flight 447 (top) along with numerous geological formations. 
One possible implementation is to resurvey a set number of anomalies per track line. This provides the operator with a richer data product while keeping the overall mission complexity low, simplifying tracking, and improving operator trust in the autonomy. Background classes can be saved prior to deployment and subsequently ignored, or anomalies can be transmitted acoustically to allow an operator to decide whether or not a resurvey is merited. Even in the complete absence of human feedback, anomalies can serve as useful navigation landmarks, providing a stationary and unique reference for the AUV to reset its accumulated navigation error.

\section{CONCLUSION}

Many of the greatest advances in autonomy over the past few decades, from unmanned aerial drones to commercial airline autopilot systems, have borne the most fruit when autonomy is used to enhance operator perception rather than fully replacing human oversight (Mindell, 2015). However, this paradigm is particularly difficult in underwater robotics due to the extreme limitations of the communications channel, which slow not only the feedback loop of meaning and trust between vehicle and operator but also the time constant for development. One of the challenges facing developers of marine autonomy is to design behaviors that, while they may inherently occur unpredictably, still prove tractable to human operators in the field. As a result, we advocate for parallel development of co-robotic approaches that blur the traditional lines between AUVs and ROVs and hasten the cycles of meaning, trust, and development while creating smarter and more precise tools for understanding the ocean. C

\section{REFERENCES}

Farr, N., A. Bowen, J. Ware, C. Pontbriand, and M. Tivey. 2010. An integrated, underwater optical/acoustic communications system. In Proceedings of OCEANS 2010 MTS/IEEE. Seattle, September 20-23, 2010.

Freitag, L., M. Grund, S. Singh, J. Partan, P. Koski, and K. Ball. 2005. The WHOI micro-modem: An acoustic communications and navigation system for multiple platforms. In Proceedings of OCEANS 2005 MTS/IEEE. Washington, DC, September 17-23, 2005.

Girdhar, Y., and G. Dudek. 2010. ONSUM: A system for generating online navigation summaries. In Proceeding of the 2010 IEEE/RSJ International Conference on Intelligent Robots and Systems. Taipei, Taiwan, October 18-22, 2010, https://doi.org/10.1109/IROS.2010.5650315.

Kaeli, J.W., and H. Singh. 2015. Online data summaries for semantic mapping and anomaly detection with autonomous underwater vehicles. In Proceedings of MTS/IEEE OCEANS 2015. Genoa, Italy, May 18-21, 2015, https://doi.org/10.1109/ OCEANS-Genova.2015.7271577.

Kaeli, J.W. 2016. Real-time anomaly detection in side-scan sonar imagery for adaptive AUV missions. Pp. 85-89 in Proceedings of 2016 IEEE-OES Autonomous Underwater Vehicles (AUV). Tokyo, Japan, November 6-9, 2016, https://doi.org/10.1109/AUV.2016.7778653.

Mindell, D.A. 2015. Our Robots, Ourselves: Robotics and the Myths of Autonomy. Viking, $260 \mathrm{pp}$.

Murphy, C., and H. Singh. 2010. Wavelet compression with set partitioning for low bandwidth telemetry from AUVs. In Proceedings of the Fifth ACM International Workshop on Underwater Networks. Woods Hole, Massachusetts, September 30-October 1, 2010, http://wuwnet.acm.org/2010/papers/004.pdf.

\section{AUTHOR}

Jeffrey W. Kaeli (jkaeli@whoi.edu) is Research Engineer, Applied Ocean Physics \& Engineering Department, Woods Hole Oceanographic Institution, Woods Hole, MA, USA.

\section{ARTICLE CITATION}

Kaeli, J.W. 2017. Do AUVs dream of electric eels? Oceanography 30(2):169-171, https://doi.org/10.5670/oceanog.2017.239. 